\title{
Analyse de minimisation de coût des fournitures utilisées pour la préparation et l'administration d'une dose d'antinéoplasique en établissement de santé
}

\author{
par Annaelle Soubieux, Caroline Plante, Johann-François Ouellette-Frève, Audrey Chouinard et \\ Jean-François Bussières
}

\section{RÉSUMÉ}

Contexte : Il existe de nombreuses stratégies visant à réduire les risques d'exposition professionnelle aux médicaments dangereux qui pèsent sur les travailleurs du domaine de la santé, dont les systèmes utilisés pour la préparation et l'administration des médicaments.

Objectif : L'objectif principal était de comparer le coût des fournitures utilisées pour la préparation et l'administration d'une dose d'antinéoplasique par voie intraveineuse (IV) dans un établissement de santé canadien pour adultes entre un système classique et un autre intégrant un système clos de transfert de médicament (SCTM).

Méthode : Il s'agit d'une analyse de minimisation de coûts. La perspective adoptée est celle d'un établissement de santé universitaire type. Lévaluation ne porte que sur le coût des fournitures utilisées pour la préparation et l'administration d'une dose d'antinéoplasique IV. Il n'est pas nécessaire de procéder à l'actualisation des coûts. Nous avons déterminé 12 scénarios comportant certaines des 11 étapes possibles de la préparation et de l'administration d'une dose IV d'antinéoplasique.

Résultats : Le coût des fournitures utilisées pour la préparation et l'administration d'une dose d'antinéoplasique varie entre 9,89 \$ et 22,37 \$ la dose pour le système classique et entre 12,34 \$ et 64,19 \$ la dose pour les systèmes intégrant un SCTM. Le surcoût moyen annuel des systèmes intégrant un SCTM est de 1,63 à 3,15 fois supérieur par rapport au système classique et il représente une dépense annuelle additionnelle allant de 363566 \$ à 1238072 \$ par année pour un établissement de santé type pour adultes.

Conclusion : Cette analyse de minimisation de coûts présente des données originales entourant la préparation et l'administration IV d'antinéoplasiques. Compte tenu des coûts importants associés à la préparation et l'administration des antinéoplasiques, les décideurs devraient mener des analyses complètes des coûts et des conséquences afin d'assurer une prise de décision éclairée.

Mots-clés : médicaments dangereux, préparation, administration, système clos de transfert de médicament

J. Can. Pharm. Hosp. 2020;73(1):27-36

\begin{abstract}
Background: Many strategies aim to reduce the risk of work-related exposure to hazardous drugs for health care workers; these strategies include the use of specific systems to prepare and administer these drugs.

Objective: To compare the cost of supplies used for preparing and administering one IV dose of antineoplastic in an adult health care facility in Canada between the traditional approach and one using a closed-system drug transfer device (CSTD).

Method: This study was a cost reduction analysis conducted from the perspective of a typical university health care facility. The assessment focused only on the cost of supplies used to prepare and administer one IV dose of antineoplastic. It was not necessary to account for discounting. We developed 12 scenarios involving some of the 11 possible steps in preparing and administering one IV dose of antineoplastic.

Results: The cost of supplies used to prepare and administer one IV dose of antineoplastic ranged between $\$ 9.89$ and $\$ 22.37$ per dose with the classical system, and between $\$ 12.34$ and $\$ 64.19$ per dose for systems involving a CSTD. The annual average extra cost of systems involving a CSTD was 1.63 to 3.15 higher than the cost with the classical system and represents extra spending of between \$363566 and \$1 238072 each year for a typical adult health care institution.

Conclusion: This cost reduction analysis presents original data relating to the preparation and administration of IV antineoplastics. Given the significant costs associated with preparing and administering antineoplastic drugs, decision-makers should perform a thorough analysis of costs and consequences to allow informed decisions to be made.
\end{abstract}

Keywords: hazardous drug, preparation, administration, closed-system drug transfer device 


\section{INTRODUCTION}

$\mathrm{D}$ epuis plusieurs années déjà, on reconnaît le danger que courent les travailleurs de la santé exposés aux médicaments dangereux ${ }^{1}$. Ces derniers sont définis par le National Institute of Occupational Safety and Health (NIOSH) comme comportant une des caractéristiques suivantes : cancérogène, tératogène, génotoxique, toxique pour la reproduction, toxique pour un organe à faible dose. Ils sont catégorisés en trois groupes (groupe 1 : antinéoplasiques, groupe 2 : les médicaments non antinéoplasiques répondant à au moins une des caractéristiques du $\mathrm{NIOSH}$, groupe 3 : représentant un risque pour la reproduction) $)^{2}$. Les travailleurs de la santé peuvent être exposés à ces risques à différentes étapes du circuit du médicament, tant à la pharmacie que dans les zones de traitement des patients ${ }^{3-5}$.

Plusieurs lignes directrices encadrent la préparation et l'administration des antinéoplasiques et des autres médicaments dangereux afin de diminuer les risques d'exposition des travailleurs. Elles comportent différentes stratégies, dont le choix de fournitures destinées à la préparation et à l'administration des médicaments ${ }^{1,6-13}$.

Plusieurs fournitures sont nécessaires aux différentes étapes du circuit du médicament, dont des systèmes classiques ou des systèmes intégrant un système clos de transfert de médicaments (SCTM). Les SCTM sont des " dispositifs de transfert de médicaments qui empêchent mécaniquement le transfert de contaminants de l'environnement dans le système et la fuite du médicament dangereux ou des concentrations de vapeurs dangereuses vers l'extérieur du système $»^{1}$. Les SCTM different des systèmes classiques en permettant une connexion sèche lors des transferts de médicaments (p. ex. adaptation aux fioles, aux seringues, aux tubulures). Ils empêcheraient ainsi la formation d'aérosols ou de gouttelettes de médicaments dangereux et protégeraient mieux le personnel des expositions à ces médicaments. Il n'existe pas de protocole universel d'évaluation de l'efficacité des SCTM $^{14}$. Cependant, vu le risque d'exposition et de contamination croisée, quelques sociétés savantes recommandent l'utilisation de SCTM pour la préparation et l'administration des médicaments dangereux ${ }^{6,10}$.

Certaines études ont démontré des réductions de contamination des surfaces à la suite de l'implantation de SCTM à la pharmacie ${ }^{15-19}$. Toutefois, une méta-analyse du groupe Cochrane dit qu'à «l'heure actuelle, aucune conclusion définitive ne peut être tirée quant à l'effet des SCTM associés à une manipulation sans danger par rapport à une manipulation sans danger sans SCTM en raison de preuves de très faible qualité fournies par les principales études évaluées. Des essais randomisés, contrôlés et multicentriques sont réalisables en fonction de la proportion de personnes exposées. Les futures études devraient également évaluer l'exposition à une sélection pertinente de médicaments dangereux utilisés à l'hôpital et mesurer les effets directs sur la santé à court terme $»^{20}$. Comme le soulignent les auteurs de la revue Cochrane, une série chronologique interrompue permettrait une évaluation dans un contexte réel en utilisant un protocole de recherche quasi expérimental.

Il existe en outre très peu d'études économiques portant sur les coûts associés à la préparation et à l'administration des doses d'antinéoplasiques avec des systèmes intégrant des SCTM ${ }^{21-23}$. Compte tenu des différents types de SCTM disponibles, des coûts additionnels associés à l'utilisation de ces fournitures spécifiques et des ressources disponibles en santé, il nous semble utile de comparer le coût des fournitures utilisées pour la préparation et l'administration de doses d'antinéoplasiques en établissement de santé.

\section{MÉTHODE}

\section{Type d'évaluation}

Il s'agit d'une analyse de minimisation de coûts. Les données sont rapportées selon les critères $\mathrm{CHEERS}^{24}$ et les lignes directrices de l'évaluation économique des technologies de la santé25.

\section{Objectif}

Lobjectif principal était de calculer le coût des fournitures utilisées pour la préparation et l'administration d'une dose d'antinéoplasique avec un système classique et un système intégrant un SCTM. L'objectif secondaire était de comparer le coût d'un système classique à celui d'un sytème intégrant différents SCTM disponibles au Canada et d'estimer le coût annuel moyen dans un établissement de santé universitaire type.

\section{Population cible}

L'analyse cible les fournitures utilisées pour la préparation et l'administration de doses d'antinéoplasiques par voie IV en établissement de santé pour une patientèle adulte ( $\geq 18$ ans).

\section{Perspective}

La perspective retenue est celle de l'établissement de santé universitaire type.

\section{Comparateur}

Il existe différentes techniques de préparation et d'administration des médicaments dangereux, qui nécessitent des fournitures différentes (p. ex. purge de la tubulure à la pharmacie ou dans les services de soins, arbres d'administration différents selon les établissements de santé). Dans cette étude, nous avons choisi d'analyser les systèmes classiques et les systèmes intégrant un SCTM pour la préparation et l'administration de doses d'antinéoplasiques en tenant compte des exigences en vigueur ${ }^{11,13}$, des recommandations des membres du groupe d'experts sur les médicaments dangereux de l'ASSTSAS et d'observations directes. Nous avons déterminé les SCTM disponibles au Canada en date du $1^{\text {er juillet } 2018 .}$ 


\section{Horizon temporel}

Il s'agit d'une analyse transversale des coûts en date du $1^{\text {er }}$ septembre 2018.

\section{Actualisation}

L'évaluation ne porte que sur le coût des fournitures utilisées pour la préparation et l'administration d'une dose d'antinéoplasique IV. Il n'est pas nécessaire de procéder à l'actualisation des coûts.

\section{Modélisation des scénarios de préparation et d'administration}

Il existe différentes options pour préparer et administrer un médicament dangereux. Les fioles peuvent avoir des diamètres différents, le médicament peut être une poudre à reconstituer ou une solution prête à l'emploi. Les médicaments dangereux peuvent être administrés en seringues ou en sacs et le rinçage peut être différent selon les habitudes de chaque établissement de santé (p. ex. rétrograde ou à partir d'une seringue de rinçage). En fonction de ces différents choix, les fournitures à utiliser sont différentes. Nous avons ainsi déterminé 12 scénarios pouvant inclure certaines des 11 étapes possibles de la préparation et de l'administration d'une dose IV d'antinéoplasique (tableau 1). Ces scénarios découlent des caractéristiques des contenants commerciaux de médicaments disponibles au Canada, des différentes fournitures disponibles au Canada, des protocoles et procédures cliniques et de 11 prémisses:

1. Diamètre des fioles - Le diamètre varie entre $13 \mathrm{~mm}$,

$20 \mathrm{~mm}$ et $28 \mathrm{~mm}$; le diamètre des fioles peut faire varier les fournitures utilisées (p. ex. adaptateurs de fioles de taille différente pour la préparation); seuls les contenants de 13 et $20 \mathrm{~mm}$ ont été retenus (les établissements de santé sondés n'utilisent pas ceux de $28 \mathrm{~mm}$ ).

2. Fiole utilisée par dose - Les doses varient pour plusieurs raisons (p. ex. indication, poids, surface corporelle, état clinique, protocole); nous avons considéré qu'une seule fiole par dose était utilisée (soit, pour un patient). Une fiole préparée $=$ une dose.

3. Préparation à partir d'une fiole de $13 \mathrm{~mm}$ - Il n'existe pas d'adaptateur classique (spike) pour le prélèvement de doses dans les fioles de $13 \mathrm{~mm}$; il en existe toutefois pour les SCTM. Une aiguille a été retenue pour le prélèvement d'une dose dans le système classique et un adaptateur pour le système intégrant un SCTM.

4. Préparation à partir d'une fiole de $20 \mathrm{~mm}$ - Un adaptateur classique ou SCTM a été retenu pour le prélèvement d'une dose.

5. Préparation de la dose - Une seringue de $10 \mathrm{~mL}$ a été retenue pour la préparation de la dose.

6. Amorçage de la tubulure à la pharmacie — L'amorçage était effectué à la pharmacie.

7. SCTM - adaptateur pour la dilution / le prélèvement à partir d'une fiole - Nous avons retenu un adaptateur avec un ballon et un adaptateur sans ballon, lorsque cela était possible.

8. SCTM - adaptateur avec une seringue "classique " - Nous avons retenu, en fonction de la disponibilité, l'adaptateur avec une seringue " classique " et non avec une seringue intégrée (dispositif SCTM fusionné avec une seringue spécifique).

Tableau 1. Douze scénarios pour la préparation et l'administration d'une dose intraveineuse d'antinéoplasique dans un établissement de santé québécois

Étapes

\begin{tabular}{|c|c|c|c|c|c|c|c|c|c|c|}
\hline \multicolumn{4}{|c|}{ Diluer / prélever } & \multirow{2}{*}{$\begin{array}{c}\text { Compléter } \\
\text { le } \\
\text { condition- } \\
\text { nement } \\
\text { de la } \\
\text { seringue } \\
\text { (contenant } \\
\text { final) } \\
\text { (étape 5) }\end{array}$} & \multirow{2}{*}{$\begin{array}{l}\text { Injecter } \\
\text { le } \\
\text { contenu } \\
\text { dans un } \\
\text { contenant } \\
\text { final (sac) } \\
\text { (étape 6) }\end{array}$} & \multicolumn{2}{|c|}{$\begin{array}{c}\text { Relier et administrer } \\
\text { le contenant } \\
\text { final au patient } \\
\text { (sac ou seringue) } \\
\text { Serinque Sac }\end{array}$} & \multicolumn{3}{|c|}{$\begin{array}{l}\text { Retirer le contenant } \\
\text { final du patient }\end{array}$} \\
\hline $\begin{array}{c}\text { Poudre } \\
13 \mathrm{~mm} \\
\text { (étape 1) }\end{array}$ & $\begin{array}{c}\text { Poudre } \\
20 \mathrm{~mm} \\
\text { (étape 2) }\end{array}$ & $\begin{array}{c}\text { Solution } \\
13 \mathrm{~mm} \\
\text { (étape 3) }\end{array}$ & $\begin{array}{c}\text { Solution } \\
20 \mathrm{~mm} \\
\text { (étape 4) }\end{array}$ & & & $\begin{array}{l}\text { Seringue } \\
\text { (étape 7) }\end{array}$ & $\begin{array}{c}\text { Sac } \\
\text { (étape 8) }\end{array}$ & $\begin{array}{l}\text { Seringue } \\
\text { (étape 9) }\end{array}$ & $\begin{array}{l}\text { Sac rincé } \\
\text { en } \\
\text { rétrograde } \\
\text { (étape 10) }\end{array}$ & $\begin{array}{c}\text { Sac } \\
\text { rincé } \\
\text { avec } \\
\text { une } \\
\text { seringue } \\
\text { (étape 11) }\end{array}$ \\
\hline
\end{tabular}

Scénario

\begin{tabular}{|c|c|c|c|c|c|c|c|c|c|c|c|}
\hline 1 & $x$ & & & & $x$ & & $x$ & & $x$ & & \\
\hline 2 & & $x$ & & & $x$ & & $x$ & & $x$ & & \\
\hline 3 & & & $x$ & & $x$ & & $x$ & & $x$ & & \\
\hline 4 & & & & $x$ & $x$ & & $x$ & & $x$ & & \\
\hline 5 & $x$ & & & & & $x$ & & $x$ & & $x$ & \\
\hline 6 & $x$ & & & & & $x$ & & $x$ & & & $x$ \\
\hline 7 & & $x$ & & & & $x$ & & $x$ & & $x$ & \\
\hline 8 & & $x$ & & & & $x$ & & $x$ & & & $x$ \\
\hline$\overline{9}$ & & & $x$ & & & $x$ & & $x$ & & $x$ & \\
\hline 10 & & & $x$ & & & $x$ & & $x$ & & & $x$ \\
\hline 11 & & & & $x$ & & $x$ & & $x$ & & $x$ & \\
\hline 12 & & & & $x$ & & $x$ & & $x$ & & & $x$ \\
\hline
\end{tabular}


9. SCTM - adaptateur avec une tubulure " classique " - Nous avons retenu l'adaptateur (bag spike) avec l'utilisation de tubulure "classique " et non avec une tubulure intégrée (dispositif SCTM fusionné avec une une seringue spécifique).

10. Administration de la dose - dispositifs - Aucune aiguille n'a été utilisée pour le couplage du contenant final et de la tubulure au patient.

11. Rinçage après l'administration — Le rinçage avec injection d'une solution compatible avec une seringue et une aiguille pour le système classique ne semblait pas sécuritaire. Tous les rinçages étaient effectués de manière rétrograde pour le système classique. Le rinçage était effectué en injectant une solution compatible directement dans le sac d'antinéoplasique avec une seringue intégrant un SCTM ou de manière rétrograde pour les systèmes intégrant un SCTM.

\section{Modélisation du coût annuel}

Trois chefs de département de pharmacie de centres hospitaliers universitaires pour adultes au Québec ont été sondés sur les données de la consommation et les méthodes d'administration de toutes les fioles d'antinéoplasiques utilisées pour la préparation et l'administration IV de doses pendant l'exercice financier de 2017-2018. Les établissements choisis ont un volume important de préparations de médicaments dangereux. Ils sont représentatifs des centres universitaires pour adultes du Québec. La consommation moyenne de ces établissements a servi à estimer les coûts moyens par année et par dose de préparation et d'administration d'antinéoplasique IV dans un établissement de santé universitaire type.

\section{Efficacité clinique}

Aux fins de notre analyse, tous les SCTM ont été considérés équivalents entre eux en termes d'efficacité.

\section{Mesure et évaluation de la santé}

Notre étude de minimisation de coûts n'a pas tenu compte des retombées potentielles de l'utilisation des différentes fournitures considérées sur la santé des travailleurs, compte tenu de l'absence de preuves de leur effet sur la santé20.

\section{Utilisation et coût des ressources}

L'étude a porté sur les coûts d'acquisition des fournitures utilisées spécifiquement pour la préparation et l'administration de dose d'antinéoplasique par voie IV. Toutefois, les coûts d'acquisition de certains produits complémentaires, similaires entre les SCTM et les systèmes classiques, n'ont pas été pris en compte (p. ex. diluants, solutés primaires, secondaires, contenant final utilisé pour le médicament antinéoplasique à moins qu'il ne s'agisse de la seringue utilisée pour la préparation) dont les choix varient selon les pratiques de chaque établissement. Les coûts d'incinération ont été pris en compte car les SCTM sont des dispositifs volumineux. Les coûts d'aménagement (p. ex. locaux), de formation, de gestion (p. ex. approvisionnement), de temps de manipulation par le personnel de la pharmacie et le personnel soignant, de formation ou de certification du personnel n'ont pas été évalués.

Les coûts d'acquisition des fournitures pour le système classique étaient ceux provenant de l'entente d'approvisionnement en commun des fabricants à contrat avec SigmaSanté (groupement d'achat des hôpitaux Québécois) en date du 1/9/2018, tandis que les coûts des SCTM ont été fournis par écrit par les fabricants selon les prix en vigueur au moment de la demande. Les fabricants ont été prévenus que les coûts seraient publiés et ils ont signé un consentement.

Les coûts d'incinération ( $\$ \mathrm{CA} / \mathrm{kg})$ en date du $1^{\mathrm{er}}$ septembre 2018 ont également été pris en compte.

Tous les prix sont en dollars canadiens (\$CA).

\section{Analyse}

Dans un premier temps, le profil des coûts d'acquisition des fournitures retenues pour la préparation et l'administration d'antinéoplasiques a été établi par système (chaque SCTM et classique). Les fournitures ont été pesées sans emballage et sans liquide à l'intérieur pour permettre le calcul du coût d'incinération. À partir de ces coûts et des scénarios établis, les investigateurs ont calculé le coût et le poids par dose pour la préparation et l'administration d'antinéoplasique par le système.

Dans un deuxième temps, un sondage effectué auprès des trois chefs de département de pharmacie a servi à l'élaboration du profil d'un établissement universitaire type. Puis les investigateurs ont procédé au calcul du nombre total moyen de fioles utilisées par établissement et par année. Ils ont également quantifié la proportion moyenne de fioles par diamètre et la proportion moyenne de fioles destinées à des doses administrées en seringues ou en sacs. Ces données ont permis d'établir la proportion de chacun des 12 scénarios applicables à un établissement type pour adultes.

Dans un troisième temps, l'estimation du coût annuel des fournitures par système pour un établissement type donné a pris en considération le coût par dose de chaque scénario en fonction des proportions du profil de l'établissement universitaire type. Le calcul du coût moyen par dose a été basé sur le coût annuel par système, soit en divisant le coût annuel par système par le nombre moyen de doses par année de l'établissement universitaire type. De plus, le calcul du surcoût des systèmes intégrant un SCTM par rapport au système classique résulte de la division du coût annuel estimé du système intégrant un SCTM par le coût annuel estimé du système classique. Compte tenu que le rinçage avec injection d'une solution compatible à l'aide d'une seringue et d'une aiguille n'est pas jugé sécuritaire pour le système classique, tous les surcoûts ont été calculés en divisant le coût annuel estimé 
du système intégrant un SCTM par le coût annuel estimé du système classique avec rinçage rétrograde.

Dans un quatrième temps, l'estimation du poids annuel des fournitures par système pour un établissement universitaire type donné résulte du poids par dose de chaque scénario en fonction des proportions du profil d'établissement universitaire type. Le coût moyen annuel d'incinération par système provient de la multiplication de ce poids annuel moyen par le coût de l'incinération.

\section{Incertitude}

Bien que les fournitures utilisées pour la préparation et l'administration de doses d'antinéoplasiques soient jetables, elles peuvent néanmoins être réutilisées (p. ex. l'adaptateur de fiole peut servir à prélever la dose supplémentaire d'un patient, la tubulure peut être installée, selon les pratiques de l'établissement, pour une période pouvant aller de 24 à 96 heures) lorsque cela est applicable. Une analyse complémentaire a permis de tenir compte de la préparation et de l'administration d'une $2^{\mathrm{e}}$ et d'une $3^{\mathrm{e}}$ dose en réutilisant les fournitures nécessaires, lorsque cela était possible.

Les investigateurs ont en outre procédé au calcul des statistiques descriptives (moyenne, écart-type).

\section{RÉSULTATS}

\section{Comparateur}

Sept systèmes intégrant des SCTM provenant de quatre fabricants ont été déterminés comme étant disponibles sur le marché canadien en date du $1^{\mathrm{er}}$ septembre 2018 : Phaseal, Phaseal Optima et Texium (Becton Dickinson), Chemoclave et Chemolock (ICU Medical), Equashield (Equashield) et Onguard (B. Braun). Les sept systèmes intégrant des SCTM ont été comparés au système classique défini pour l'étude.

\section{Coût de préparation et d'administration d'une dose d'antinéoplasique dans un établissement de santé et comparaison des coûts selon les SCTM évalués}

L'annexe 1 (disponible au https://www.cjhp-oline.ca/ index.php/cjhp/issue/view/195/showToc) présente le coût et le poids des fournitures retenues pour la préparation et l'administration d'une dose IV d'antinéoplasique en établissement de santé par un système classique. Certaines de ces fournitures conviennent également aux systèmes intégrant un SCTM (p. ex. tubulures, seringues).

L'annexe 2 (disponible au https://www.cjhp-online.ca/ index.php/cjhp/issue/view/195/showToc) présente le coût des fournitures retenues pour la préparation et l'administration par un système intégrant un SCTM d'une dose IV d'antinéoplasique en établissement de santé.

En ce qui concerne le système classique, le coût des fournitures de préparation et d'administration d'une dose IV d'antinéoplasique variait entre 9,89 \$ et 22,37 \$ la dose selon les 12 scénarios (annexes 3 et 4, disponibles au https://www.cjhponline.ca/index.php/cjhp/issue/view/195/showToc).

En ce qui concerne le système intégrant un SCTM, le coût des fournitures de préparation et d'administration d'une dose IV d'antinéoplasique variait entre $12,34 \$$ et 64,19 la dose pour les 12 scénarios évalués (annexes 3 et 4 ).

\section{Profil de l'établissement de santé universitaire type}

Chaque établissement utilisait en moyenne, 32 767ะ11 234 fioles d'antinéoplasiques par année. La majorité des fioles, soit 80,9 \% (26 509/32 767), avaient un diamètre de $20 \mathrm{~mm}$ et les autres, 19,1\%, avaient un diamètre de 13 mm (6 256/32 767). La majorité des doses IV, soit 81,6\%, étaient administrées en sacs (26 742/32 767), alors que l'administration des autres, soit 18,4\%, l'était en seringues (6 024/32 767). Les scénarios 11 et 12 représentaient la majorité des doses utilisées dans un établissement de santé universitaire type (47,8 \%) (tableau 2). Nous avons regroupé les scénarios qui incluent un rinçage rétrograde et par seringue.

\section{Estimation du coût annuel}

L'estimation du coût annuel des fournitures du système classique pour la préparation et l'administration des doses IV d'antinéoplasiques dans un établissement de santé universitaire type se montait à 574778 \$. L'estimation du coût annuel des fournitures du système intégrant des SCTM pour la préparation et l'administration des doses IV d'antinéoplasiques dans un établissement de santé universitaire type variait entre 938346 \$ et 1812861 \$ (Figure 1).

\section{Tableau 2. Répartition du nombre de fioles par scénario retenu}

\begin{tabular}{|c|c|c|c|c|c|c|c|c|c|}
\hline \multirow{3}{*}{$\begin{array}{l}\text { Paramètre } \\
\text { Nombre moyen } \\
\text { de fioles par } \\
\text { établissement } \\
\text { par année }\end{array}$} & \multicolumn{9}{|c|}{ Scénario } \\
\hline & 1 & 2 & 3 & 4 & 5 et $6 *$ & 7 et $8^{*}$ & 9 et $10^{*}$ & 11 et $12^{*}$ & Total \\
\hline & 1314 & 1496 & 853 & 2361 & 1552 & 6973 & 2538 & 15679 & 32767 \\
\hline Ëcart-type & 578 & 721 & 508 & 1694 & 808 & 3311 & 1373 & 5429 & 11234 \\
\hline Proportion (\%) & 4 & 4,6 & 2,6 & 7,2 & 4,7 & 21,3 & 7,8 & 47,8 & 100 \\
\hline
\end{tabular}



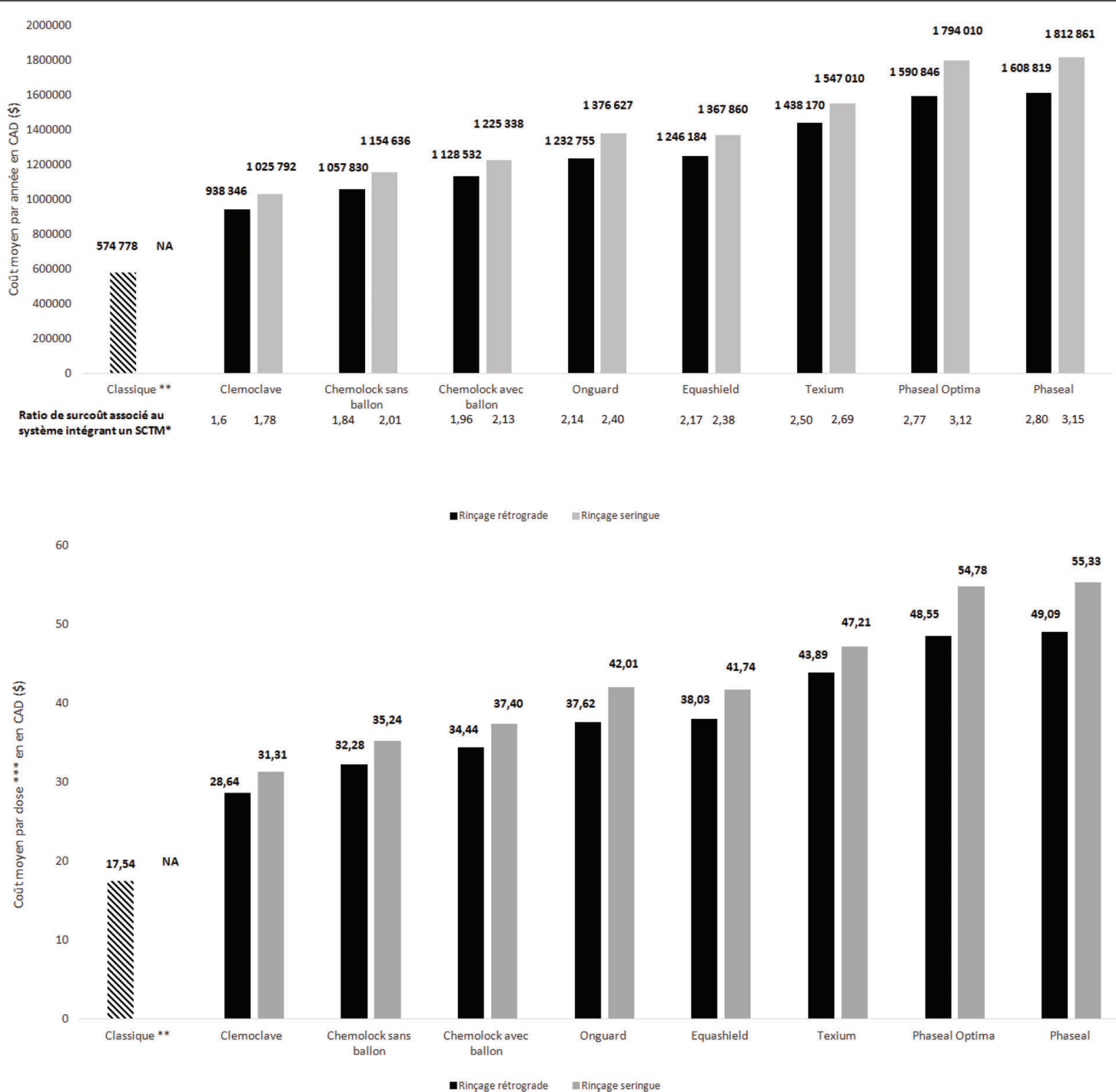

Figure 1. Coûts moyens par année et par dose ainsi que le surcoût associé à un système intégrant un système "clos de transfert de médicament (SCTM) pour un établissement de santé type. *Le surcoût des systèmes intégrant un SCTM par rapport au système classique a été calculé en divisant le coût annuel estimé du système intégrant un SCTM en particulier par le coût annuel estimé du système classique applicable. Puisque le rinçage avec la seringue est jugé non sécuritaire pour le système classique, tous les surcoûts ont été calculés en divisant par le coût annuel estimé du système classique avec rinçage rétrograde. **Pour le système classique, le rinçage à l'aiguille est jugé non sécuritaire. Noté NA (non applicable). ${ }^{* * *}$ Le coût moyen par dose a été calculé en divisant le coût moyen par année par le nombre moyen de dose par année.

\section{Incinération des fournitures utilisées}

En date du $1^{\text {er }}$ septembre 2018, le coût d'incinération des déchets des antinéoplasiques était de 1,07 \$/kg selon l'entente en vigueur à Sigmasanté. Pour un établissement universitaire type, le poids annuel des fournitures du système classique était de $4190 \mathrm{~kg}$, ce qui représente un coût moyen annuel d'incinération de 4 483,30 \$. De plus, le poids annuel des fournitures des systèmes intégrant un SCTM (comprenant des fournitures classiques applicables) variait entre 4655 et $5622 \mathrm{~kg}$, ce qui représente un coût moyen annuel d'incinération situé entre 4 980,85 \$ et $6015,54 \$$. 


\section{Incertitude}

Les trois scénarios les plus représentatifs de la pratique dans un établissement de santé universitaire type ont servi à réaliser l'analyse d'incertitude, soit l'administration en seringues (scénario 4) et en sacs (scénarios 11 et 12). Le coût des fournitures nécessaires à la préparation et à l'administration d'une, de deux ou de trois doses d'antinéoplasiques par système pour les trois scénarios ciblés varie entre 3,49 \$ et 17,44 \$ par dose pour le système classique et entre $6,32 \$$ et $60,69 \$$ par dose pour un système intégrant un SCTM (tableau 3).

\section{DISCUSSION}

À notre connaissance, il s'agit de la première analyse de minimisation des coûts de préparation et d'administration

\section{Tableau 3. Analyse d'incertitude - Coût par dose de la préparation et de l'administration d'une, de deux ou de trois doses par système pour trois scénarios ciblés}

\begin{tabular}{|c|c|c|c|c|c|c|c|c|c|}
\hline \multirow[t]{2}{*}{$\begin{array}{l}\text { Système et } \\
\text { variables }\end{array}$} & \multicolumn{3}{|c|}{$\begin{array}{l}\text { Préparation et administration } \\
\text { d'une dose par système }\end{array}$} & \multicolumn{3}{|c|}{$\begin{array}{l}\text { Préparation et administration } \\
\text { de deux doses par système }\end{array}$} & \multicolumn{3}{|c|}{$\begin{array}{l}\text { Préparation et administration } \\
\text { de trois doses par système }\end{array}$} \\
\hline & Scénario 4 & Scénario 11 & Scénario12 & Scénario 4 & Scénario11 & Scénario12 & Scénario 4 & Scénario11 & Scénario12 \\
\hline \multicolumn{10}{|l|}{ Classique } \\
\hline $\begin{array}{l}\text { Nombre d'objets } \\
\text { requis* }\end{array}$ & 4 & 7 & NA $\neq$ & 2 & 4 & NAł & 2 & 4 & NAł \\
\hline Coût par doset & 9,89 & 17,44 & NAł & 5,09 & 9,38 & NAł & 3,49 & 6.68 & NAł \\
\hline \multicolumn{10}{|l|}{ Chemoclave } \\
\hline $\begin{array}{l}\text { Nombre d'objets } \\
\text { requis* }\end{array}$ & $5 \S$ & 11 & 13 & $3 \S$ & 6 & 8 & $3 \S$ & 6 & 8 \\
\hline Coût par doset & 12,34 & 30,82 & 34.09 & 7,82 & 20,08 & 23,35 & 6,32 & 16,50 & 19,77 \\
\hline \multicolumn{10}{|c|}{ Chemolock sans ballon } \\
\hline $\begin{array}{l}\text { Nombre d'objets } \\
\text { requis* }\end{array}$ & 6 & 12 & 14 & 3 & 6 & 8 & 3 & 6 & 8 \\
\hline Coût par doset & 15,14 & 34,47 & 38,09 & 9,40 & 22,68 & 26,30 & 7,48 & 18,75 & 22,37 \\
\hline \multicolumn{10}{|l|}{ Onguard } \\
\hline $\begin{array}{l}\text { Nombre d'objets } \\
\text { requis* }\end{array}$ & 5 & 11 & 13 & 2 & 5 & 7 & 2 & 5 & 7 \\
\hline Coût par doset & 18,24 & 40,50 & 45,88 & 11,70 & 27,52 & 32,90 & 9,52 & 23,20 & 28,58 \\
\hline \multicolumn{10}{|c|}{ Chemolock avec ballon } \\
\hline $\begin{array}{l}\text { Nombre d'objets } \\
\text { requis* }\end{array}$ & 6 & 12 & 14 & 3 & 6 & 8 & 3 & 6 & 8 \\
\hline Coût par doset & 18,59 & 37,92 & 41,54 & 11,12 & 24,41 & 28,03 & 8,63 & 19,90 & 23,52 \\
\hline \multicolumn{10}{|l|}{ Equashield } \\
\hline $\begin{array}{l}\text { Nombre d'objets } \\
\text { requis* }\end{array}$ & 6 & 12 & 14 & 3 & 6 & 8 & 3 & 6 & 8 \\
\hline Coût par doset & 19,67 & 40,68 & 45,23 & 12,73 & 27,26 & 31,81 & 10,41 & 22,79 & 27,34 \\
\hline \multicolumn{10}{|l|}{ Texium } \\
\hline $\begin{array}{l}\text { Nombre d'objets } \\
\text { requis* }\end{array}$ & 4 & 9 & 11 & 2 & 5 & 7 & 2 & 5 & 7 \\
\hline Coût par doset & 26,75 & 44,79 & 48,86 & 15,30 & 28,20 & 32,27 & 11,49 & 22,67 & 26,74 \\
\hline \multicolumn{10}{|l|}{ Phaseal Optima } \\
\hline $\begin{array}{l}\text { Nombre d'objets } \\
\text { requis* }\end{array}$ & 5 & 11 & 13 & 2 & 5 & 7 & 2 & 5 & 7 \\
\hline Coût par doset & 24,35 & 52,50 & 60,13 & 15,88 & 36,01 & 43,64 & 13,06 & 30,51 & 38,13 \\
\hline \multicolumn{10}{|l|}{ Phaseal } \\
\hline $\begin{array}{l}\text { Nombre d'objets } \\
\text { requis* }\end{array}$ & 6 & 12 & 14 & 3 & 6 & 8 & 3 & 6 & 8 \\
\hline Coût par doset & 24,91 & 53,06 & 60,69 & 16,41 & 36,54 & 44,17 & 13,58 & 31,03 & 38,66 \\
\hline \multicolumn{10}{|c|}{$\begin{array}{l}\text { NA = non applicable, coût par dose est en \$CA. } \\
\text { *Le nombre d'objets requis pour deux ou trois doses correspond aux fournitures nécessaires et non réutilisables dès la première dose } \\
\text { (p. ex. seringue). } \\
\text { tLe coût par dose pour la deuxième dose est obtenu en additionnant les coûts d'acquisition des fournitures nécessaires (réutilisables et comptées } \\
\text { une seule fois ainsi que non réutilisables et comptées deux fois) divisés par deux pour ramener à un coût par dose. Le même principe est appliqué } \\
\text { pour la troisième dose. } \\
\text { fLe rinçage par la seringue avec une aiguille est jugé non sécuritaire pour le système classique. } \\
\text { §Exemple du calcul du nombre d'objets pour le SCTM Chemoclave selon le scénario } 4 \text { (Fiole de } 20 \text { mm en solution, préparée pour une administra- } \\
\text { tion en seringue): Dose 1, utilisation de } 5 \text { objets - Un adaptateur de fiole SCTM (réutilisation pour les autres doses), une seringue classique } \\
\text { (non-réutilisation pour les autres doses), un adaptateur de seringue SCTM (non réutilisable pour les autres doses), un bouchon SCTM à mettre } \\
\text { sur la seringue pour le transport dans le service (non réutilisable pour les autres doses), une tubulure d'administration classique reliée au patient, } \\
\text { permettant de passer la seringue (réutilisation pour une autre dose au même patient). Pour cet exemple, on n'a pas besoin d'adaptateur SCTM } \\
\text { raccordé à l'embout de la tubulure où sera placée la seringue, car la technologie du SCTM Chemoclave s'adapte à toutes les connexions Luer Lock. } \\
\text { Ce n'est pas le cas de tous les SCTM (p. ex. Phaseal et Phaseal optima, Equashield, Onguard, Chemolock). Dose 2, utilisation de } 3 \text { objets - une } \\
\text { seringue classique, un adaptateur de seringue, un bouchon à mettre sur la seringue. Dose 3, utilisation de } 3 \text { objets - une seringue classique, } \\
\text { un adaptateur de seringue, un bouchon à mettre sur la seringue. }\end{array}$} \\
\hline
\end{tabular}


d'antinéoplasiques qui compare des systèmes intégrant les SCTM au Canada. L'estimation du coût annuel des fournitures pour la préparation et l'administration des doses d'antinéoplasiques dans un établissement de santé universitaire type induit par le système intégrant des SCTM variait entre 938346 \$ et 1812861 \$, ce qui représente un coût moyen situé entre $28,64 \$$ et $55,32 \$$ par dose selon le SCTM. Le surcoût moyen annuel des systèmes intégrant un SCTM par rapport au système classique variait entre 1,63 fois et 3,15 fois, soit respectivement une dépense supplémentaire minimale de 363568 \$ par année et maximale de 1238083 \$ par année. Les coûts liés aux fournitures requises pour la préparation et l'administration d'une dose IV d'antinéoplasique varient grandement. Le coût annuel moyen d'incinération des fournitures représente des frais négligeables par rapport aux coûts d'acquisition des fournitures. Si on tient compte en outre de la possibilité de préparer ou d'administrer deux ou trois doses d'antinéoplasiques avec les mêmes fournitures, les coûts peuvent varier énormément. La réutilisation des fournitures, quand cela est possible, permet de diminuer les coûts à la fois dans le groupe des systèmes classiques et dans celui des SCTM.

Une revue de la littérature publiée récemment recense seulement 12 articles présentant les coûts associés à un système intégrant un SCTM ${ }^{21}$. Ces publications étaient souvent incomplètes (moyenne de 9,2/24 critères CHEERS rapportés). Les coûts rapportés dans la littérature concernaient majoritairement le système Phaseal. Nous avons cependant répertorié sur le marché canadien sept SCTM possibles. Les coûts retrouvés dans les études sont hétérogènes et difficilement comparables (c.-à-d. devises différentes, barème de calcul des coûts variable, qui inclut le coût annuel, le coût par dose, le coût pour la préparation seulement, etc.). Mullot et collab. rapportaient une estimation des coûts annuels de $160000 €$ liés au système Phaseal pour la préparation d'environ 24400 doses d'antinéoplasiques ${ }^{26}$. Edwards et collab. rapportaient des coûts annuels de 106557 \$ US pour la pharmacie ayant recours au SCTM de type Phaseal ${ }^{27}$. Ces estimations de coûts sont inférieures à nos calculs, mais elles ne prennent pas en compte les coûts liés à l'administration des doses. Une autre étude évaluant les coûts d'acquisition liés à un système intégrant le Phaseal pour la préparation rapporte des frais de 40,92 \$ US par $\operatorname{dose}^{28}$. Ce coût par dose se rapproche davantage des 55,32 \$ par dose de notre analyse, qui représentent le coût moyen par dose d'un système intégrant Phaseal. Par ailleurs, un hôpital nordaméricain a estimé le coût annuel d'implantation d'un SCTM à 1,5 millions \$ US, ce qui se rapproche davantage des intervalles calculés dans notre étude ${ }^{22}$.

Selon le chapitre 797 de l'United States Pharmacopeia (USP), les fioles à dose unique utilisées pour les préparations stériles effectuées dans des conditions ISO5 doivent comporter une date limite d'utilisation (DLU) de six heures pour limiter le risque de contamination microbienne ${ }^{29}$. L'Association nationale des organismes de réglementation de la pharmacie (ANORP) recommande également une DLU de six heures ${ }^{10}$. L'Ordre des pharmaciens du Québec (OPQ) recommande une DLU de $24 \mathrm{~h}^{11}$. Quelques auteurs ont observé que les coûts d'acquisition associés aux systèmes intégrant un SCTM pourraient être compensés par une économie associée à la prolongation de la DLU envisagée lorsqu'on utilise un système intégrant un SCTM $^{27,30,31}$. Peu d'études ont comparé la stérilité des fioles percées à l'aide d'un sytème classique et de systèmes intégrant un SCTM $^{32-34}$. Une revue de littérature sur le sujet confirme cette lacune $e^{35}$. De plus, aucun organisme n'appuie actuellement la prolongation de la DLU avec l'utilisation de systèmes intégrant un $\mathrm{SCTM}^{6,11,22}$. En outre, une méta-analyse récente montre un faible taux de contamination $(0,08 \%)$ des fioles préparées à la pharmacie (intervalle de confiance à $95 \% 0-0$ ) et cela sans mention d'utilisation de $\mathrm{SCTM}^{36}$. Chaque établissement de santé devrait évaluer son risque de base. Il n’est peut-être pas possible de démontrer la réduction accrue de la contamination en utilisant un système intégrant un SCTM, compte tenu du très faible taux de contamination de base. La décision de prolonger la DLU devrait être discutée avec l'organisme réglementaire concerné.

Différentes mesures contribuent à limiter les risques liés à l'exposition professionnelle des travailleurs aux antinéoplasiques (p. ex. nettoyer les fioles contaminées à leur réception à la pharmacie, porter des équipements de protection individuelle, respecter les bonnes pratiques de préparation) $)^{1,7,37}$. Les SCTM font partie de ces mesures. Les professionnels de la santé et particulièrement ceux qui exercent au chevet des patients sont exposés à des traces de médicaments dangereux lors de l'administration d'une dose d'antinéoplasique. Toutefois, l'exposition aux excreta des patients est une source de contamination non négligeable qui ne peut pas être réduite par les systèmes intégrant un SCTM. Dans la revue Cochrane portant sur l'efficacité des systèmes intégrant un SCTM, les auteurs ont noté une faible réduction des traces de cyclophosphamide $\left(50 \mathrm{pg} / \mathrm{cm}^{2}\right)$ et d'épirubicine $\left(110 \mathrm{pg} / \mathrm{cm}^{2}\right)$ à la pharmacie, mais pas pour les autres antinéoplasiques étudiés, en dépit de l'utilisation de systèmes intégrant un SCTM. Cette revue systématique ne permet pas non plus de confirmer la provenance des traces (c.-à-d. contenant de médicament, tubulure, excreta) ${ }^{20}$. Ainsi, compte tenu des coûts élevés associés à l'utilisation des SCTM, la décision de les utiliser doit s'appuyer sur le recours à toutes les autres mesures de protection des travailleurs et de réduction du risque. Il convient de préciser que les systèmes i ntégrant un SCTM sont une option permettant de retirer les aiguilles pour la préparation et l'administration des doses d'antinéoplasiques par voie parentérale.

Du fait des coûts élevés associés aux systèmes intégrant un SCTM, la sélection d'un SCTM en particulier devrait prendre en compte les données probantes, l'évaluation de la mise en place adéquate des autres mesures de protection des travailleurs, l'analyse actuelle des contaminations de surface de l'établissement, une 
analyse économique comparative des pratiques actuelles et des systèmes envisagés intégrant un SCTM. Il semble également utile que la sélection d'un système intégrant un SCTM se fasse dans le cadre d'un appel d'offres régional ou national afin d'obtenir les meilleurs prix.

Dans le chapitre 800 de l'USP, les experts recommandent l'utilisation rigoureuse d'un système intégrant un SCTM pour l'administration de doses d'antinéoplasiques lorsque cela est possible (critère 5.4) tout en évoquant l'utilité d'un tel système pour la préparation, sans en exiger l'usage. Il est possible de réduire les coûts associés à l'utilisation de SCTM en ne retenant que les fournitures utilisées pour l'administration des doses (c.-̀̀-d. adaptateur permettant à l'infirmière de raccorder de façon étanche une seringue ou un sac aux tubulures utilisées pour l'administration) et en excluant les fournitures spécifiques à la préparation (p. ex. adaptateur de fiole). Notre analyse ne comporte pas de tels scénarios.

Cette analyse de minimisation de coûts ne cible que les fournitures utilisées pour la préparation et l'administration d'une dose IV d'antinéoplasique en date du $1^{\text {er }}$ septembre 2018. Dans cette étude, une fiole correspond à une dose pour un patient, ce qui représente une analyse prudente, car une fiole peut être utilisée pour d'autres doses destinées à d'autres patients. Il est également possible que plusieurs fioles soient nécessaires pour un même patient. Une future analyse devrait prendre en compte le nombre de doses préparées par un établissement et non le nombre de fioles afin que l'analyse de la consommation soit plus fine. Pour les fins de cette analyse, nous avons interrogé trois établissements de santé qui sont représentatifs des établissements universitaires du Québec, mais qui ne reflètent pas à eux seuls les pratiques de la province. De plus, l'analyse ne tient pas compte des coûts liés aux infrastructures, à l'achat de médicaments, à l'utilisation d'autres fournitures, aux ressources humaines et de gestion. D'autres scénarios pourraient être envisagés, compte tenu des pratiques locales. Lanalyse ne tient pas compte des avantages et des inconvénients associés à chaque système. Chaque fabricant de SCTM met en valeur les avantages de ses produits et il existe des différences entre les systèmes qui peuvent influencer les préférences des utilisateurs en termes d'ergonomie et de temps de manipulation. Compte tenu des différents concepts de SCTM, des données publiées et de l'absence d'un protocole valide permettant de comparer l'efficacité de chaque SCTM, nous avons choisi de prendre en considération les systèmes équivalents. D'autres travaux pourraient comparer les coûts associés aux manipulations des divers SCTM (c.-à-d. temps-mouvements).

\section{CONCLUSION}

Le système classique engendre des coûts de fournitures nécessaires à la préparation et à l'administration d'une dose d'antinéoplasique qui varient entre 9,89 \$ et 22,37 \$ la dose et les coûts des systèmes intégrant un SCTM se situent entre 12,34 \$ et 64,19 \$ la dose. Le surcoût moyen annuel des systèmes intégrant un SCTM par rapport au système classique est de 1,63 fois à 3,15 fois supérieur et il représente une dépense annuelle supplémentaire de 363566 \$̀ 1238072 \$ pour un établissement de santé universitaire type pour adultes. Compte tenu des coûts importants associés à la préparation et à l'administration des antinéoplasiques, les décideurs devraient mener des analyses complètes des coûts et des conséquences afin de prendre des décisions éclairées.

\section{Références}

1. NIOSH Alert: Preventing occupational exposures to antineoplastic and other hazardous drugs in health care. Cincinnati $(\mathrm{OH})$ : Department of Health and Human Services (États-Unis), Centers for Disease Control and Prevention, National Institute for Occupational Safety and Health; 2004. Publié au : https://www.cdc.gov/niosh/docs/2004-165/pdfs/2004-165.pdf. Consultéle $1^{\mathrm{er}}$ avril 2019.

2. Connor TH, MacKenzie BA, DeBord DG, Trout DB, O'Callaghan JP. $\mathrm{NIOSH}$ list of antineoplastic and other hazardous drugs in healthcare settings, 2016. Cincinnati $(\mathrm{OH})$ : Department of Health and Human Services (États-Unis), Centers for Disease Control and Prevention, National Institute for Occupational Safety and Health; 2016. DHHS (NIOSH) publication no 2016-161. Publié au : https:/www.cdc.gov/niosh/docs/ 2016-161/pdfs/2016-161.pdf. Consulté le $1^{\text {er }}$ avril 2019.

3. Hedmer M, Georgiadi A, Bremberg ER, Jönsson BA, Eksborg S. Surface contamination of cyclophosphamide packaging and surface contamination with antineoplastic drugs in a hospital pharmacy in Sweden. Ann Occup Hyg. 2005;49(7):629-37.

4. Fleury-Souverain S, Nussbaumer S, Mattiuzzo M, Bonnabry P. Determination of the external contamination and cross-contamination by cytotoxic drugs on the surfaces of vials available on the Swiss market. J Oncol Pharm Pract. 2014;20(2):100-11.

5. Connor TH, Sessink PJM, Harrison BR, Pretty JR, Peters BG, Alfaro RM, et al. Surface contamination of chemotherapy drug vials and evaluation of new vial-cleaning techniques: results of three studies. Am J Health Syst Pharm. 2005;62(5):475-84.

6. General chapter $<800>$ : Hazardous drugs - handling in healthcare settings. Dans : USP 40-NF 35, second supplement. Bethesda (MD): US Pharmacopeial Convention (USP); 2017. Publié au : http://www.usp.org/ sites/default/files/usp/document/our-work/healthcare-quality-safety/generalchapter-800.pdf. Consulté le $1^{\text {er }}$ avril 2019.

7. Power L, Coyne J. ASHP guidelines on handling hazardous drugs. Am J Health Syst Pharm. 2018;75(24):1996-2031.

8. Bernabeu-Martínez M, Ramos Merino M, Santos Gago J, Álvarez Sabucedo L, Wanden-Berghe C, Sanz-Valero J. Guidelines for safe handling of hazardous drugs: a systematic review. PLoS One. 2018;13(5):e0197172.

9. Easty A, Coakley N, Cheng R, Cividino M, Savage P, Tozer R, et al. Safe handling of cytotoxics: guideline recommendations. Curr Oncol. 2015; 22(1):e27-37.

10. Model standards for pharmacy compounding of hazardous sterile preparation. Ottawa (ON) : National Association of Pharmacy Regulatory Authorities; 2016. Publié au : https://napra.ca/sites/default/files/2017-09/Mdl_Stnds_ Pharmacy_Compounding_Hazardous_Sterile_Preparations_Nov2016_ Revised_b.pdf. Consulté le $1^{\text {er }}$ avril 2019.

11. Norme 2014.02. Préparation de produits stériles dangereux en pharmacie. Montréal (QC) : Ordre des pharmaciens du Québec; 2014. Publié au https://www.opq.org/doc/media/1847_38_fr-ca_0_norme201402_prod_ striles_dang_oct2017.pdf. Consulté le $1^{\text {er }}$ avril 2019.

12. Guide de prévention : Manipulation sécuritaire des médicaments dangereux. Montréal (QC) : ASSTSAS [Association paritaire pour la santé et la sécurité du travail du secteur affaires sociales]. Publié au : http://asstsas.qc.ca/sites/ default/files/publications/documents/Guides_Broch_Depl/GP65_ medicaments_dangereux.pdf. Consulté le $1^{\mathrm{er}}$ avril 2019.

13. Méthode de soins infirmiers - administration d'agents antinéoplasiques par voie intraveineuse. Québec (QC) : Ministère de la santé et des services sociaux, Direction générale de cancérologie; 2018. Publié au : http://publications. msss.gouv.qc.ca/msss/fichiers/2018/18-902-11W.pdf. Consulté le $1^{\text {er }}$ avril 2019.

14. A performance protocol for closed system transfer devices used during pharmacy compounding and administration of hazardous drugs [brouillon] 
This single copy is for your personal, non-commercial use only.

For permission to reprint multiple copies or to order presentation-ready copies for distribution, contact CJHP at publications@cshp.ca

Cincinnati $(\mathrm{OH})$ : National Institute for Occupational Safety and Health; 2015. Publié au : https://www.cdc.gov/niosh/docket/review/docket288a/ pdfs/aperformancetestprotocolforclosedsystemtransferdevices.pdf. Consulté le $1^{\text {er }}$ avril 2019.

15. Harrison BR, Peters BG, Bing MR. Comparison of surface contamination with cyclophosphamide and fluorouracil using a closed-system drug transfer device versus standard preparation techniques. Am J Health Syst Pharm. 2006;63(18):1736-44

16. Sessink PJM, Connor TH, Jorgenson JA, Tyler TG. Reduction in surface contamination with antineoplastic drugs in 22 hospital pharmacies in the US following implementation of a closed-system drug transfer device. J Oncol Pharm Pract. 2011;17(1):39-48.

17. Sessink PJM, Trahan J, Coyne JW. Reduction in surface contamination with cyclophosphamide in 30 US hospital pharmacies following implementation of a closed-system drug transfer device. Hosp Pharm. 2013;48(3):204-12.

18. Simon N, Vasseur M, Pinturaud M, Soichot M, Richeval C, Humbert L, et al. Effectiveness of a closed-system transfer device in reducing surface contamination in a new antineoplastic drug-compounding unit: a prospective, controlled, parallel study. PloS One. 2016;11(7):e0159052.

19. Miyake T, Iwamoto T, Tanimura M, Okuda M. Impact of closed-system drug transfer device on exposure of environment and healthcare provider to cyclophosphamide in Japanese hospital. SpringerPlus. 2013;2(1):273.

20. Gurusamy KS, Best LM, Tanguay C, Lennan E, Korva M, Bussières JF. Closed-system drug-transfer devices plus safe handling of hazardous drugs versus safe handling alone for reducing exposure to infusional hazardous drugs in healthcare staff. Cochrane Database Syst Rev. 2018;3:CD012860.

21. Soubieux A, Tanguay C, Lachaine J, Bussières JF. Review of economic data on closed system transfer drug for preparation and administration of hazardous drugs. Eur J Hosp Pharm. 2019. doi: 10.1136/ejhpharm-2018001775. [EPUB avant impression].

22. Furlow B. How to improve the safety of chemotherapy administration. Oncol Nurse Advisor. 2010;:21-5.

23. Lowry F. Closed-system transfer devices lessen worker contamination-at a cost. Pharm Pract News. 2009;:36.

24. Husereau D, Drummond M, Petrou S, Carswell C, Moher D, Greenberg D, et al. Consolidated Health Economic Evaluation Reporting Standards (CHEERS) — explanation and elaboration: a report of the ISPOR Health Economic Evaluation Publication Guidelines Good Reporting Practices Task Force. Value Health. 2013;16(2):231-50.

25. Lignes directrices de l'évaluation économique des technologies de la santé au Canada. $4^{e}$ édition. Ottawa $(\mathrm{ON})$ : ACMTS (Agence canadienne des médicaments et des technologies de la santé); 2017. Publié au : https:// www.cadth.ca/sites/default/files/pdf/guidelines_for_the_economic_ evaluation_of_health_technologies_canada_4th_ed_f.pdf. Consulté le $1^{\text {er }}$ avril 2019 .

26. Mullot H, Blondeel S, Escalup L, Negellen S, Chenailler C, Pelloquin A, et al. Intérêt et faisabilité des systèmes Tevadaptor ${ }^{\circledR}$ et Phaseal ${ }^{\circledR}$ dans une unité centralisée de préparation des anticancéreux. Pharm Hosp. 2008;43(175): 189-99.

27. Edwards MS, Solimando DA Jr, Grollman FR, Pang JL, Chasick AH, Hightman CM, et al. Cost savings realized by use of the PhaSeal ${ }^{\circledR}$ closed-system transfer device for preparation of antineoplastic agents. J Oncol Pharm Pract. 2013;19(4):338-47.

28. Chan HK, Lim YM. Cost analysis of using a closed-system transfer device (CSTD) for antineoplastic drug preparation in a Malaysian governmentfunded hospital. Asian Pac J Cancer Prev. 2016;17(11):4951-7.

29. < $797>$ Pharmaceutical compounding — sterile preparations. Bethesda (MD): US Pharmacopeial Convention; 2008. Publié au : https:/www.sefh.es/ fichadjuntos/USP797GC.pdf. Consulté le $1^{\text {er }}$ avril 2019.
30. Rowe EC, Savage SW, Rutala WA, Weber DJ, Gergen-Teague M, Eckel SF. Economic and microbiologic evaluation of single-dose vial extension for hazardous drugs. J Oncol Pharm Pract. 2012;8(4):45-9.

31. Juhász Á, Batka G, Szucs A. Responding to drug shortages and rising costs: IV chemotherapy drug use optimization achieved by closed safety devices in hospital pharmacies. Drugs Ther Perspect. 2016;32(4):170-6.

32. Whitehead MC, Vanetten CL, Jacob ME, Harrison TM. Microbial integrity of preservative-free alfaxalone in a multiple-use system for two storage conditions and three handling techniques. Am J Vet Res. 2018;79(7):704-10.

33. Savry A, Correard F, Bennis Y, Roubaud S, Gauthier-Villano L, Pisano P, et al. Aseptic simulation test for cytotoxic drug production in isolators. Am J Health Syst Pharm. 2014;71(6):476-81.

34. Garrigue P, Montana M, Ventre C, Savry A, Gauthier-Villano L, Pisano P, et al. Safe cytotoxic drug preparation using closed-system transfer device: technical and practical evaluation of a new device (Vialshield/Texium) comparatively to a reference one (Phaseal). Int J Pharm Compound. 2016; 20(2):148-54

35. Soubieux A, Tanguay C, Bussières JF. Review of studies examining microbial contamination of vials used for preparations done with closed-system drug transfer devices. Eur J Hosp Pharm. 2019. doi 10.1136/ejhpharm-2019001913. [EPUB avant impression].

36. Larmené-Beld KHM, Frijlink HW, Taxis K. A systematic review and meta-analysis of microbial contamination of parenteral medication prepared in a clinical versus pharmacy environment. Eur J Clin Pharmacol. 2019; 75(5):609-17.

37. Nygren O, Gustavsson B, Ström L. Cisplatin contamination observed on the outside of drug vials. Ann Occup Hyg. 2002;46:555-7.

Annaelle Soubieux travaille à l'Unité de recherche en pratique pharmaceutique, Département de pharmacie, Centre hospitalie universitaire Sainte-Justine, Montréal (Québec). Elle est aussi candidate au D. Pharm. à l'Université Paris Descartes.

Caroline Plante, B. Sc. Inf., travaille au Département d'hémato-oncologie, Centre hospitalier universitaire Sainte-Justine, Montréal (Québec).

Johann-François Ouellette-Frève, D. Pharm., M. Sc., travaille à I'Unité de recherche en pratique pharmaceutique, Département de pharmacie, Centre hospitalier universitaire Sainte-Justine, Montréal (Québec).

Audrey Chouinard, M. Sc. Inf., travaille à la Direction des soins infirmiers, Centre hospitalier de I'Université de Montréal, Montréal (Québec).

Jean-François Bussières, B. Pharm., M. Sc., MBA, FCSHP, FOPQ, travaille à I'Unité de recherche en pratique pharmaceutique, Département de pharmacie, Centre hospitalier universitaire Sainte-Justine, et à la Faculté de pharmacie, Université de Montréal, Montréal (Québec).

Conflits d'intérêt : Aucun déclaré.

\section{Adresse de correspondance :}

Jean-François Bussières

Département de pharmacie et Unité de recherche en pratique pharmaceutique

Centre hospitalier universitaire Sainte-Justine

3175, chemin de la Côte Sainte-Catherine

Montréal QC H3T 1C5

Courriel : jean-francois.bussieres.hsj@ssss.gouv.qc.ca

Financement : Aucun reçu 